\title{
Investigation of a New Waveguide Structure Based on Negative Index Material for Optoelectronic Applications
}

\author{
Nour El Houda Hissi ${ }^{1}$, Bouchra Mokhtari ${ }^{1 *}$, Saida Bahsine ${ }^{1}$, Noureddine Cherkaoui Eddeqaqi ${ }^{1}$, \\ Mohammed Musa Shabat ${ }^{2}$
}

${ }^{1}$ Physics Department, Moulay Ismail University, Meknes, Morocco

${ }^{2}$ Physics Department, Islamic University of Gaza, Gaza, Palestine

Email: *Nicet2012@hotmail.com

How to cite this paper: Hissi, N. El H., Mokhtari, B., Bahsine, S., Eddeqaqi, N.C. and Shabat, M.M. (2017) Investigation of a New Waveguide Structure Based on Negative Index Material for Optoelectronic Applications. Optics and Photonics Journal, 7, 123-132.

https://doi.org/10.4236/opj.2017.77012

Received: May 23, 2017

Accepted: July 11, 2017

Published: July 14, 2017

Copyright (๑) 2017 by authors and Scientific Research Publishing Inc. This work is licensed under the Creative Commons Attribution International License (CC BY 4.0).

http://creativecommons.org/licenses/by/4.0/ (c) (i) Open Access

\begin{abstract}
In this work, a waveguide structure consisting of a new artificial negative index material (NIM) surrounded by a nonlinear cover and a ferrite (YIG) substrate has been designed and investigated. We apply the boundary conditions and impose the condition of negative effective permeability of the ferrite slab to derive the dispersion relation related to the proposed structure. The NIM permittivity and permeability are not constant and depend on the operating frequency. The dispersion properties of the nonlinear electromagnetic surface waves (NEM) are analyzed and the associated propagation index is calculated. Results show that the dispersion could be tuned and controlled by selecting the NIM film thickness and the film-cover interface nonlinearity. The proposed structure is supporting unusual types of NEM surface waves having a non-reciprocal behavior widely used in designing optoelectronic devices.
\end{abstract}

\section{Keywords}

Negative Index Material, Nonlinearity, Ferrite Substrate, Waveguide, Dispersion Relation, Non-Reciprocal Behavior

\section{Introduction}

Negative index materials (NIM) are artificially designed structures with negative permittivity and permeability providing a route to create potential devices with fascinating electromagnetic properties that cannot be obtained with natural materials [1]-[6]. The history of these materials began with Veselago [7] who predicted the existence of such materials with unexpected optical properties. One particularly interesting NIM device is an NIM based waveguide structure that has potentially interesting applications. Recent experimental demonstrations of 
novel composite materials with a negative refractive index [8]-[13] open up an exceptional possibility to design novel types of devices where electromagnetic waves propagate in a nonconventional way. In parallel, more linear and nonlinear metamaterials have been studied theoretically and experimentally from microwave to optical frequencies [14]-[19]. In most investigations dealing with the planar nonlinear waveguides, the basic attention has been given to the electromagnetic surface waves [20]. Despite these advanced studies, new modes of propagation due to the variation of NIM's parameters (permittivity and permeability) in new NIM waveguide were not investigated. In this paper, we aim at studying a magnetic structure with a negative index material (NIM) core surrounded by a nonlinear cover cladding and a ferrite substrate, where unusual electromagnetic surface waves-basically not existing in a conventional waveguide-are examined [21]. The present work is an extension and an integration of the previous work [12] [21]. We focus on studying the considered structure and calculate the dispersion equations for TE modes. We present and discuss the associated propagation wave index and the film cover interface nonlinearity versus the normalized frequency and other various physical parameters of the NIM layer. The numerical results are given in order to draw attention on the variation in surface wave's behavior propagating in different waveguide structures, as in the considered waveguide based on NIM core [13] [22] having negative permittivity $\varepsilon_{2}$ and permeability $\mu_{2}$ both depending on the operating frequency $\omega$, the NEM waves propagate in a non-conventional way comparing with those propagating in classical structures. Finally, conclusions are given for the various results of this study. This work's results can be used in designing and fabricating microwave devices for a wide range of applications as isolators, sensors, circulators, solar cells...

\section{Proposed Waveguide Structure and Simulation Approach}

Figure 1 displays the configuration of the considered waveguide structure. We shall assume that the waveguide consists of an NIM core of width L bounded by a nonlinear cover and a gyromagnetic ferrite substrate. The waveguide is assumed to have infinite extent in the $\mathrm{x}$ and $\mathrm{y}$ directions. The dispersion equation is given for stationary TE waves only propagating in the $\mathrm{x}$-direction. A static magnetic field is applied in the y direction transverse to the direction of propagation.

The magnetic permeability tensor of the ferrite (YIG) substrate is defined as [21] where $\omega$ is the surface wave operating frequency:

$$
\begin{aligned}
& \left\|\mu_{1}\right\|=\left(\begin{array}{ccc}
\mu_{11} & 0 & i \mu_{13} \\
0 & \mu_{22} & 0 \\
-i \mu_{13} & 0 & \mu_{11}
\end{array}\right) \quad \text { where } \quad \mu_{11}=\mu_{B}\left(1+\frac{\omega_{0} \omega_{m}}{\omega_{0}^{2}-\omega^{2}}\right) \text { and } \\
& \mu_{13}=\mu_{B} \frac{\omega \omega_{m}}{\omega_{0}^{2}-\omega^{2}} \text { and } \mu_{22}=\mu_{B} . \\
& \omega_{0}=\gamma \mu_{0} H_{0} \text { is the Larmor frequency, } \omega_{m}=\gamma \mu_{0} M_{0} \text { is the magnetic frequen- } \\
& \text { cy, } \mu_{B} \text { is the background permeability, } \gamma \text { is the gyromagnetic ratio, } \mu_{0} H_{0} \text { is }
\end{aligned}
$$




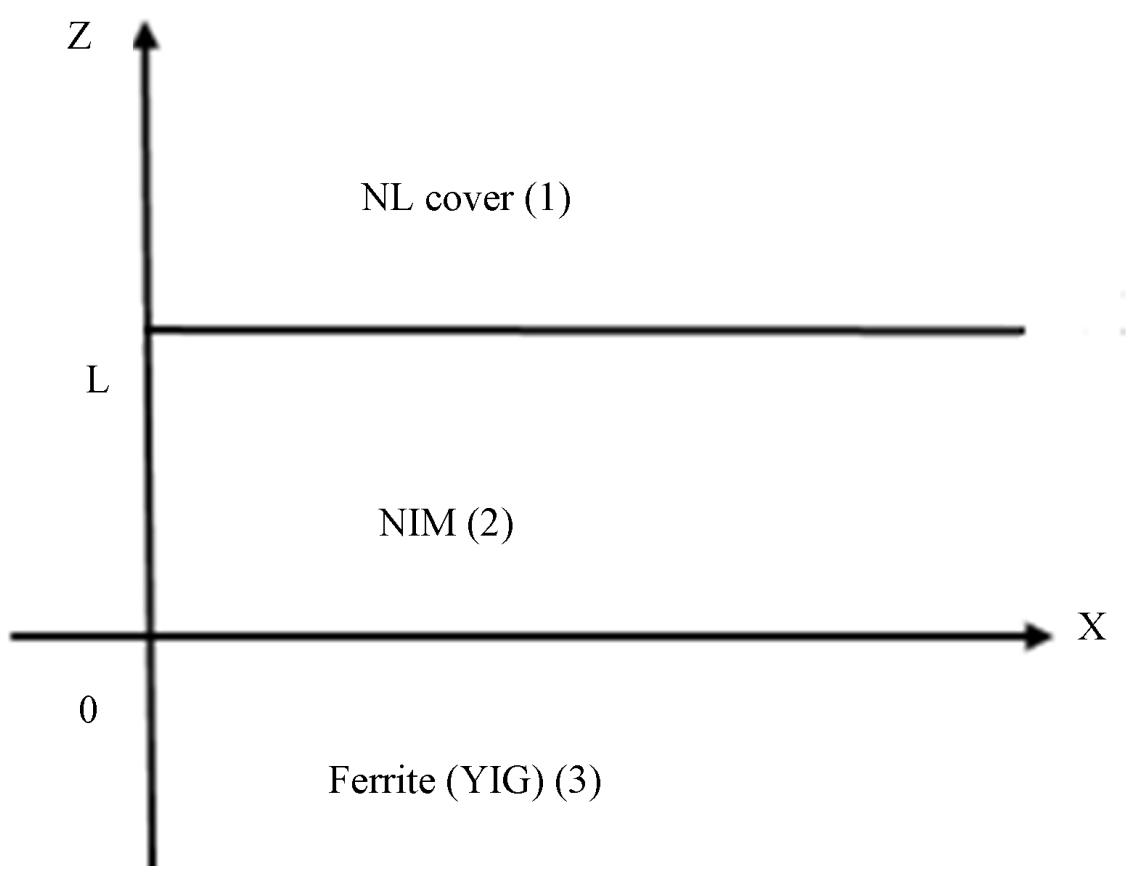

Figure 1. The schematic of the proposed waveguide consisting of negative index film bounded by a ferrite (YIG) substrate and a nonlinear (NL) cover.

the applied magnetic field and $\mu_{0} M_{0}$ is the dc saturation magnetization. The NIM core has it effective permittivity $\varepsilon_{2}(\omega)=1-\frac{\omega_{p}^{2}}{\omega^{2}}$ with the plasma frequency $\omega_{p}$ in the $\mathrm{GHz}$ range and it effective magnetic permeability $\mu_{2}(\omega)=1-\frac{F \omega^{2}}{\omega^{2}-\omega_{r}^{2}}$, with the resonance frequency $\omega_{r}$ in the GHz range; $F$ is the filling factor. We choose $\omega_{r}=4 \mathrm{GHz}$ and

$F=0.56$ [22]. The nonlinear dielectric cover has it dielectric function isotropic and depends on the electric field. It can be written as for TE waves: $\varepsilon^{N L}=\varepsilon_{f}+\alpha E_{y}^{2}$ where $\varepsilon_{f}$ is the linear part of the dielectric function and $\alpha$ is the nonlinear coefficient [21].

The TE fields have the following forms, where $\mathrm{q}$ is the propagation constant:

$$
\begin{gathered}
E=\left(0, E_{y} \mathrm{e}^{i(q x-\omega t)}, 0\right) \\
H=\left(H_{x} \mathrm{e}^{i(q x-\omega t)}, 0, H_{z} \mathrm{e}^{i(q x-\omega t)}\right)
\end{gathered}
$$

We apply the transverse electric fields into Maxwell's equations:

$$
\begin{aligned}
& \nabla \times E=i \omega \mu_{0} \mu_{i} H \\
& \nabla \times H=-i \omega \varepsilon_{0} \varepsilon_{i} E
\end{aligned}
$$

In the ferrite (YIG) substrate, the plane wave equation is:

$$
\frac{\partial^{2} E_{y}^{(1)}}{\partial z^{2}}-\tilde{\eta}_{1}^{2} E_{y}^{(1)}=0
$$

where $\tilde{\eta}_{1}^{2}=q^{2}-\frac{\omega^{2}}{c^{2}} \mu_{v} \varepsilon_{c}=\tilde{\eta}_{0}^{2}\left(\beta^{2}-\mu_{v} \varepsilon_{c}\right)$ and $\mu_{v}=\left(\mu_{11}^{2}+\mu_{13}^{2}\right) / \mu_{11}$ is the 
Voigt permeability, $\beta=\frac{q}{\tilde{\eta}_{0}}$ is the effective mode index, $\tilde{\eta}_{0}=\frac{\omega}{c}$ is the propagation constant in the vacuum, and $c$ is the speed of light.

In medium (2), the plane wave equation is:

$$
\frac{\partial^{2} E_{y}^{(2)}}{\partial z^{2}}-\tilde{\eta}_{2}^{2} E_{y}^{(2)}=0
$$

where:

$$
\tilde{\eta}_{2}^{2}=q^{2}-\frac{\omega^{2}}{c^{2}}\left(1-\frac{F \omega^{2}}{\omega^{2}-\omega_{r}^{2}}\right)\left(1-\frac{\omega_{p}^{2}}{\omega^{2}}\right)=\tilde{\eta}_{0}^{2}\left(\beta^{2}-\left(1-\frac{F \omega^{2}}{\omega^{2}-\omega_{r}^{2}}\right)\left(1-\frac{\omega_{p}^{2}}{\omega^{2}}\right)\right)
$$

In the nonlinear dielectric cover, the plane wave equation is:

$$
\frac{\partial^{2} E_{y}^{(3)}}{\partial z^{2}}-\tilde{\eta}_{3}^{2} E_{y}^{(3)}=0
$$

where:

$$
\tilde{\eta}_{3}^{2}=q^{2}-\frac{\omega^{2}}{c^{2}} \mu_{f} \varepsilon_{f}=\tilde{\eta}_{0}^{2}\left(\beta^{2}-\mu_{f} \varepsilon_{f}\right)
$$

Applying the boundary conditions at $z=0$ and $z=L$ to the plane wave equations solutions, we obtain the dispersion equation as follows:

$$
\left(\tilde{\eta}_{2}-R \mu_{2}\right)\left(\tilde{\eta}_{2}-\tilde{\eta}_{3} v \mu_{2}\right)-\left(\tilde{\eta}_{2}+R \mu_{2}\right)\left(\tilde{\eta}_{2}+\tilde{\eta}_{3} v \mu_{2}\right) \mathrm{e}^{-2 \tilde{\eta}_{2} L}=0
$$

where:

$$
R=\left(-\mu_{11} \tilde{\eta}_{1}+\mu_{13} q\right) / \mu_{11} \mu_{v} \text { and } v=\tanh \tilde{\eta}_{3}\left(z_{0}-L\right)
$$

$\mathrm{z}_{0}$ is a constant that we can determine from the boundary conditions.

From the nonlinear dispersion equation we can easily obtain the film-cover interface nonlinearity:

$$
\begin{gathered}
{\left[\frac{\alpha}{2}\right] E_{y}^{2(3)}(L)=\frac{\tilde{\eta}_{3}^{2}}{\tilde{\eta}_{0}^{2}}\left(1-v^{2}\right)} \\
v=\frac{\tilde{\eta}_{2}-\tilde{\eta}_{2} W}{\tilde{\eta}_{3} \mu_{2} W+\mu_{2} \tilde{\eta}_{3}}, \\
W=\frac{\left(\tilde{\eta}_{2}+R \mu_{2}\right) \mathrm{e}^{-2 \tilde{\eta}_{2} L}}{\tilde{\eta}_{2}-R \mu_{2}}
\end{gathered}
$$

\section{Results and Discussions}

In order to have surface waves in the proposed waveguide structure, the effective ferrite permeability should be less than zero. This constraint and condition should be implemented on the solution of the dispersion equation. We numerically solve the Equation (7) in order to find out the propagation wave index versus the operating normalized frequency $\Omega=\omega / \omega_{m}$ for different NIM film thicknesses $L$, where $\beta=c q / \omega$. The numerical computations were performed the parameter values [15]: $\mu_{0} H_{0}=0.550 T, \quad \mu_{0} M_{0}=0.1750 T, \quad \varepsilon_{c}=1$, $\varepsilon_{f}=2.25, \quad \alpha=1.55 \times 10^{-10} \mathrm{~m}^{2} \cdot \mathrm{V}^{-2}, \quad \mu_{B}=1.25$ and $\gamma=1.76 \times 10^{11} \mathrm{~s}^{-1} \cdot \mathrm{T}^{-1}$. 
Figure 2 shows the computed effective Voigt permeability $\mu_{v}$ versus the normalized operating frequency. For numerical calculation, we set the frequency within the range from $\sqrt{\omega_{0}\left(\omega_{0}+\omega_{m}\right)}$ to $\omega_{0}+\omega_{m}$ for $\mu_{v} \leq 0$.

Figure 3 shows discontinuity, that represents the forbidden band of the NIM waveguide. It clearly illustrates that the surface modes cannot propagate for $L=$ $4 \mathrm{~mm}$.

Figure 4 shows the NEM surface waves dispersion in the backward wave direction. Different NIM slab thicknesses are considered to show it effect on the propagation. The effective wave index versus the operating frequency has a new

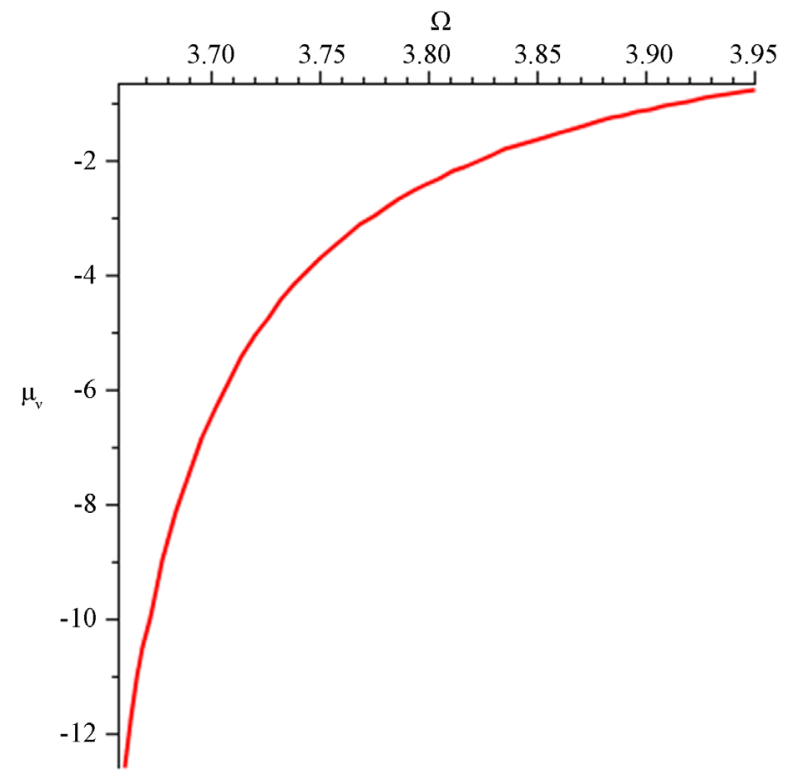

Figure 2. The effective Voigt permeability versus the normalized frequency.

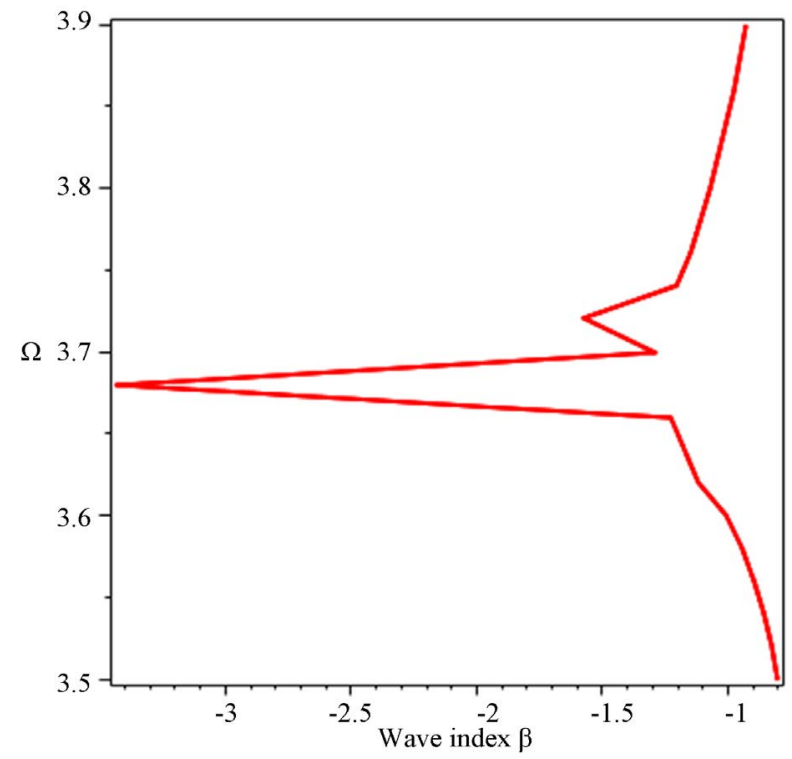

Figure 3. The dependence of $\Omega$ on the wave index for the negative NIM layer thickness $\mathrm{L}=4 \mathrm{~mm}$. 


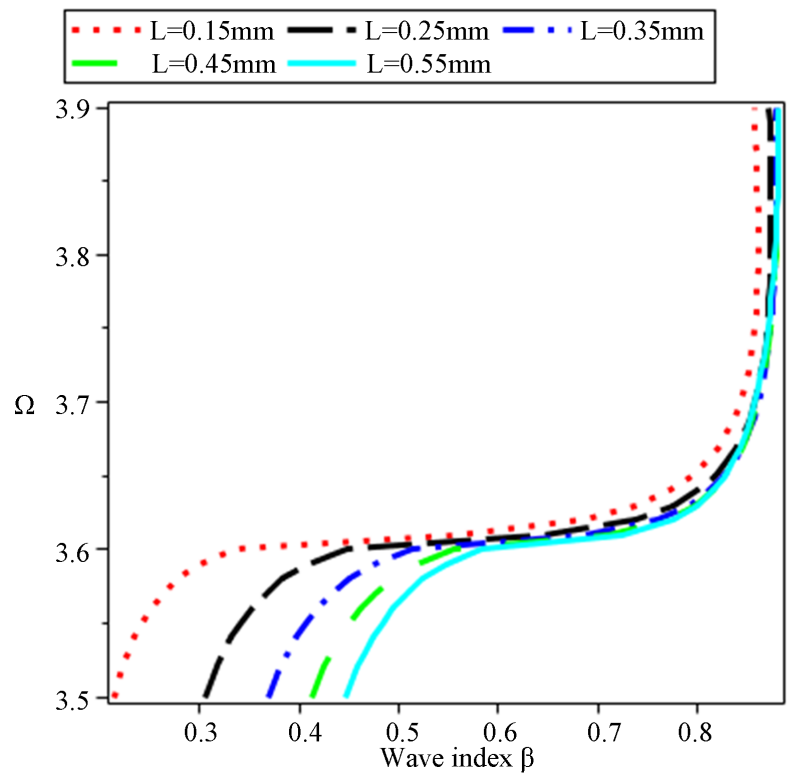

Figure 4. NEM surface wave's dispersion in the backward wave direction for different values of the NIM slabs thickness.

different behavior, we can observe that when the value of $L$ increases from 0.15 $\mathrm{mm}$ to $0.55 \mathrm{~mm}$, the wave index varies from 0.2 to 0.55 , above the value $\beta=0.6$, the propagation modes become identical and have practically the same trajectory.

Figure 5 exhibits the nonlinear surface wave's dispersion curves in the backward and the forward directions for different NIM slab thicknesses with the film cover non-linearity value kept constant. The curves present a linear increase with practically the same direction. Moreover all modes are propagating in the negative region for $\beta$ negative in the forward direction and propagating in the positive region for $\beta$ positive in the backward direction. We conclude that the NIM having it permittivity and permeability depending on the frequency is changing the forward direction to the backward direction and this behavior is particular to NIM based waveguides.

It is concluded that by adjusting or tuning some physical parameters in such waveguide structure, the wave propagation direction could be reversed. This feature or characteristics could be used in design some future optoelectronics devices.

Figure 6 shows NEM surface wave's dispersion curves in the backward and the forward directions for different NIM slab thicknesses and for a different value of nonlinearity $v=\tanh \tilde{\eta}_{3}\left(z_{0}-L\right)$ with the film cover non-linearity value kept constant. We found that two different values of the frequency correspond to the same value of the wave index; this also means that both figures show a new behavior and different stability features. This behavior is very important to design microwave devices.

The above predictions and calculations of NEM surface wave contrast behavior might be used in designing future microwave devices such as isolators and circulators. 


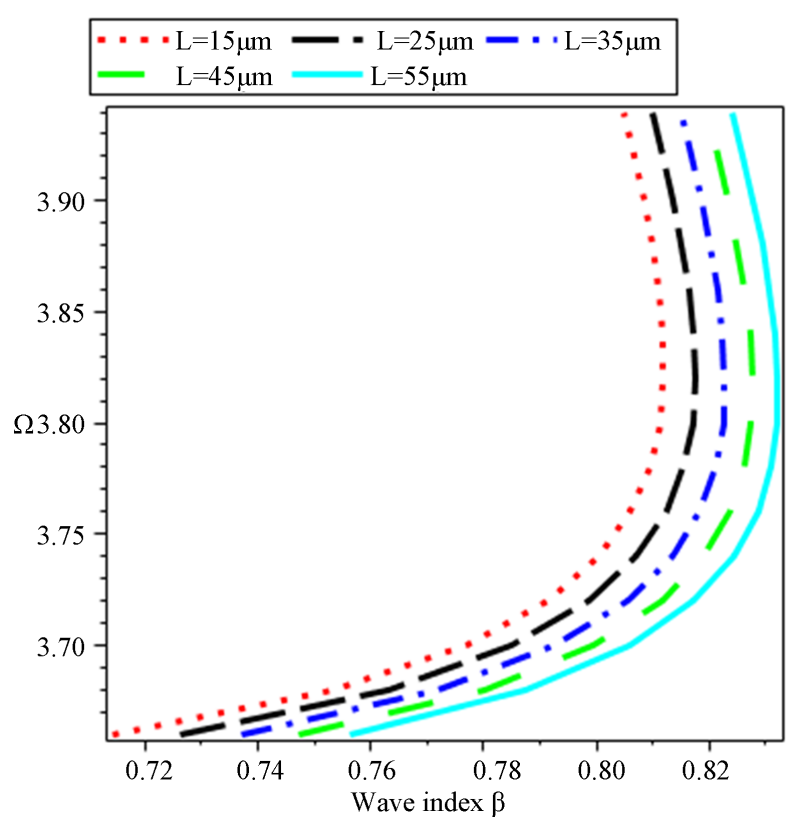

(a)

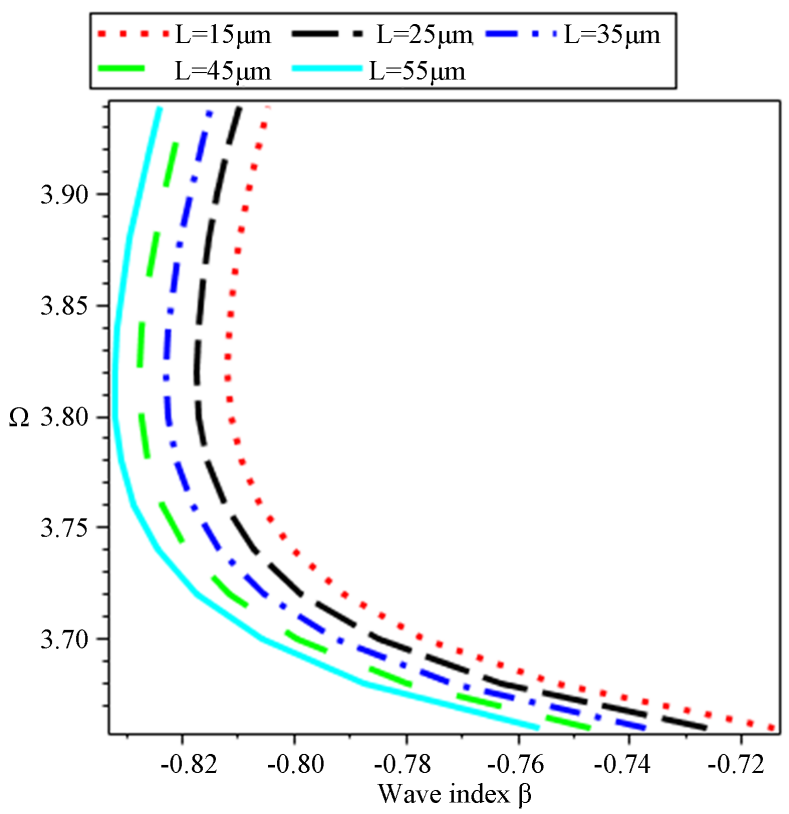

(b)

Figure 5. NEM surface wave's dispersion at $\frac{1}{2} \alpha E_{y}^{2}(L)=0.5, v=0.55$ and for different values of the NIM slab thickness (a) is the backward wave direction and (b) is forward wave direction.

\section{Conclusion}

In a three-layered structure based on NIM having it optical parameters (permittivity and permeability) depending on the operating frequency, the propagation characteristics can be controlled by selecting the NIM film thickness. The negative effect of the NIM core is reversing the forward direction of the waves to be in the backward direction. Moreover, for some values of the wave index in the 
backward propagation direction (or forward propagation direction) correspond two operating frequencies. These effects are in contrast with those obtained in a conventional waveguide structure and could be used in designing and implementing integrated microwave devices based on the non-reciprocal behavior as isolators, sensors and circulators for military services, solar cells, telecommunications, automatic access control systems and medical equipment.

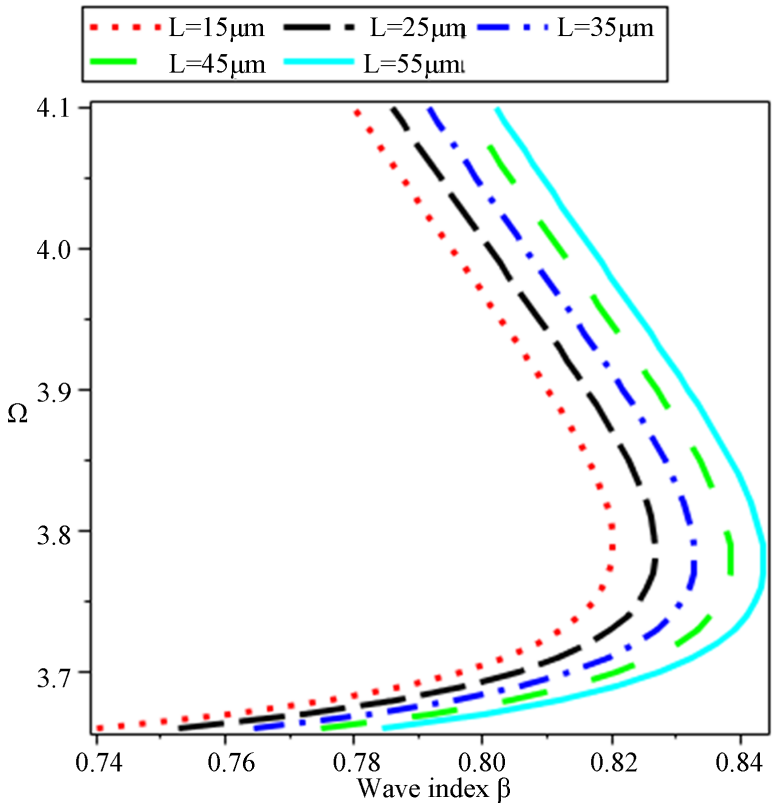

(a)

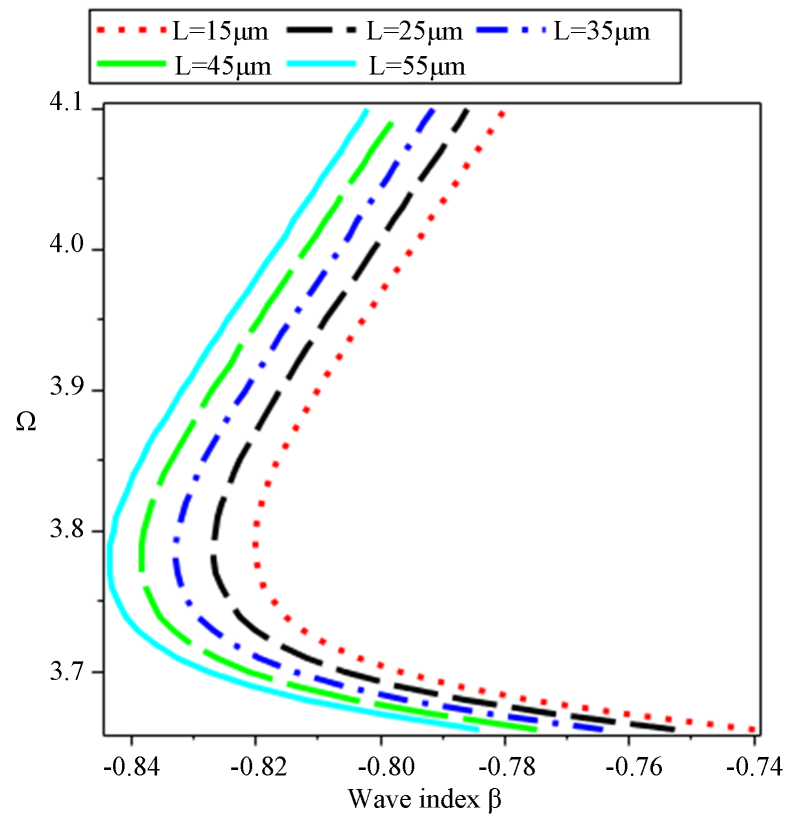

(b)

Figure 6. NEM surface wave's dispersion at $\frac{1}{2} \alpha E_{y}^{2}(L)=0.5, v=0.56$ and for different values of the thickness: $\mathrm{L}=15 \mu \mathrm{m}, \mathrm{L}=25 \mu \mathrm{m}, \mathrm{L}=35 \mu \mathrm{m}, \mathrm{L}=45 \mu \mathrm{m}, \mathrm{L}=55 \mu \mathrm{m}$. (a) is the backward wave direction and (b) is forward wave direction. 


\section{Acknowledgements}

One of the authors (M.M.S) thanks Prof. Dr. Daniel M. Schaadt, Institute of Energy Research and Physical Technologies, Technical University of Clausthal, Germany for many valuable suggestions and fruitful discussions during the development of this work.

\section{References}

[1] Wu, R.X. (2005) Effective Negative Refraction Index in Periodic Metal-FerriteMetal Film Composite. Journal of Applied Physics, 97, 076105-076108A. https://doi.org/10.1063/1.1883718

[2] Powell, D.A., Hannam, K., Shadrivov, I.V. and Kivshar, Y.S. (2011) Near-Field Interaction of Twisted Split-Ring Resonators. Physical Review B, 83, 1-6. https://doi.org/10.1103/PhysRevB.83.235420

[3] Kapitanova, P.V., Slobozhnanyuk, A.P., Shadrivov, I.V., Belov, P.A. and Kivshar, Y.S. (2012) Competing Nonlinearities with Metamaterials. Applied Physics Letters, 101, 1-4. https://doi.org/10.1063/1.4768945

[4] Zhanyuka, P.S., Kapitanovaa, P.V., Shadrivova, I.V., Belova, P.A. and Kivshara, Y. S. (2012) Metamaterials with Tunable Nonlinearity. Journal of Theoretical and Experimental Physics Letters, 95, 613-617. https://doi.org/10.1134/S0021364012120156

[5] Thapa, K.B., Vishwakarma, A., Singh, R. and Ojha, S.P. (2010) Electromagnetic Wave Propagation through Single Negative Index Material. Journal of Ovonic Research, 6, 105-115.

[6] El-Amassi, D.M., El-Khozondar, H.J. and Shabat, M.M. (2015) Efficiency Enhancement of Solar Cell Using Metamaterials. International Journal of Nano Studies and Technology, 4, 84-87. https://doi.org/10.19070/2167-8685-1500016

[7] Veselago, V.G. (1968) The Electrodynamics of Substances with Simultaneously Negative Values ofe and $\mu$. Soviet Physics Uspekhi, 10, 509-514. https://doi.org/10.1070/PU1968v010n04ABEH003699

[8] Shadrivov, I.V., Zharova, N.A., Zharov, A.A. and Kivshar, Y.S. (2004) Defect Modes and Transmission Properties of Left-Handed Band Gaps Structures. Physical Review E, 70, 1-6. https://doi.org/10.1103/PhysRevE.70.046615

[9] Shadrivov, I.V. (2004) Nonlinear Guided Waves and Symmetry Breaking in LeftHanded Waveguides. Physics Optics, 2, 175-180.

https://doi.org/10.1016/j.photonics.2004.08.003

[10] Smith, D.R., Schuring, D., Rosenblutch, M., Schultz, S., Ramakrishna, S.A. and Pendry, J.B. (2003) Limitations on Subdiffraction Imaging with a Negative Refractive Index Slab. Applied Physics Letters, 82, 1506-1508. https://doi.org/10.1063/1.1554779

[11] Darmanyan, S.A., Kobaykov, A. and Chowdhury, D.Q. (2006) Nonlinear Guided Waves in a Negative-Index Slab Waveguide. Physics Letters A, 363, 159-163. https://doi.org/10.1016/j.physleta.2006.10.087

[12] Hissi, N., Mokhtari, B., Eddeqaqi, N.C., Shabat, M.M. and Atangana, J. (2016) Nonlinear Surface Waves at Ferrite-Metamaterial Waveguide Structure. Journal of Modern. Optic, 63, 1552-1557. https://doi.org/10.1080/09500340.2016.1161094

[13] Mokhtari, B., Eddeqaqi, N.C., Atangana, J., Essama B.G.O. and Kofane, T.C. (2014) Nonlinear Dispersion Equation and Guided Modes in a Slab Waveguide Composed of a Negative-Index Medium. Optical and Quantum Electronics, 45, 155-163. 
https://doi.org/10.1007/s11082-013-9724-y

[14] Soukoulis, C.M., Liden, S. and Wegener, M. (2007) Negative Refraction Index at Optical Wavelengths. Science, 315, 47-49. https://doi.org/10.1126/science.1136481

[15] Shalaev, V.M., Cai, W., Chettiar, U.K., Yuan, H., Andrey, K., Sarychev, V., Drachev, P. and Kildishev, A.V. (2005) Negative Index of Refraction in Optical Metamaterials. Optics Letters, 30, 3356-3358. https://doi.org/10.1364/OL.30.003356

[16] Shalaev, V.M. (2007) Optical Negative-Index Metamaterials. Nature, Photonics, 1, 41-48. https://doi.org/10.1038/nphoton.2006.49

[17] Yao, J., Liu, Z., Liu, Y., Wang, Y., Sun, C., Bartal, G., Stacy, A.M. and Zhang, X. (2008) Optical Negative Refraction in Bulk Metamaterials of Nanowires. Science, 321, 930. https://doi.org/10.1126/science.1157566

[18] Zharov, A.A., Shadrivov, I.V. and Kivshar, Y.S. (2003) Nonlinear Properties of Left-Handed Metamaterials. Physical Review Letters, 91, 1-4.

https://doi.org/10.1103/PhysRevLett.91.037401

[19] Lapine, M., Gorkunov, M. and Ringhofer, K.H. (2003) Nonlinearity of a Metamaterial Arising from Diode Insertions into Resonant Conductive Elements. Physical Review E, 67, 065601. https://doi.org/10.1103/PhysRevE.67.065601

[20] El-Khozondar, H.J., Al-Sahhar, Z.I. and Shabat, M.M. (2010) Electromagnetic Surface Waves of a Ferrite Slab Bounded by Metamaterials. International Journal of Electronics and Communication, 64, 1063-1067.

https://doi.org/10.1016/j.aeue.2009.09.003

[21] Shabat, M.M. and Pelzl, J. (1996) Nonlinear Electromagnetic Surface Waves in a Magnetic Structure. Infrared Physics and Technology, 37, 265-270. https://doi.org/10.1016/1350-4495(95)00051-8

[22] Essadqui, A., Ben-Ali, J., Bria, D., Djafari-Rouhani, B. and Nougaoui, A. (2010) Photonic Band Structure of 1D Periodic Composite System with Left Handed and Right Handed Materials by Green Function Approach. Progress in Electromagnetics Research B, 23, 229-249. https://doi.org/10.2528/PIERB10032404

Scientific Research Publishing

\section{Submit or recommend next manuscript to SCIRP and we will provide best} service for you:

Accepting pre-submission inquiries through Email, Facebook, LinkedIn, Twitter, etc. A wide selection of journals (inclusive of 9 subjects, more than 200 journals)

Providing 24-hour high-quality service

User-friendly online submission system

Fair and swift peer-review system

Efficient typesetting and proofreading procedure

Display of the result of downloads and visits, as well as the number of cited articles

Maximum dissemination of your research work

Submit your manuscript at: http://papersubmission.scirp.org/

Or contactopj@scirp.org 